\title{
Bioresorbierbare Klebstoffe in der operativen
}

\section{Traumatologie und Orthopädie}

\author{
Christian Heiss, \\ Reinhard Schnettler
}

Schlagworte: Biologische/Synthetische Verbindungen Klassifikation - Klebstoffe - Bioresorbierbarer Knochenkleber

\begin{abstract}
Das Kleben ist eine attraktive Technik, zwei getrennte Materialien leicht und schnell miteinander zusammen zu fügen. Der Wunsch der Traumatologen und Orthopäden, körperfremde Materialien für diese Zwecke zu verwenden, spiegelt sich in der Entwicklung vielfältigster Surrogate wider. Die Entwicklung solcher Substanzen hat inzwischen schon eine lange Historie, ohne dass bisher eine tatsächliche klinisch einsatzfähige Alternative zumindest auf dem Gebiet der Traumatologie und Orthopädie gefunden werden konnte. Die folgenden Ausführungen sollen dem Leser einen kritischen Überblick über die verschiedenen resorbierbaren Klebstoffe verschaffen. In dem Artikel werden die nicht körpereigenen Substanzen klassifiziert und die Anforderungen an einen bioresorbierbaren Knochenklebstoff definiert. Schließlich werden Empfehlungen für den klinischen Einsatz der Verbindungen gegeben.
\end{abstract}

\section{Einleitung}

In den letzten Jahrzehnten hat der Einsatz von Klebstoffen in der Industrie bei dem Verbinden verschiedenster Materialien zugenommen und bewährte Verfahren wie das Schrauben, Nieten, Nageln, Löten und Schweißen abgelöst.

In der Medizin, vor allem in der operativen Traumatologie und Orthopädie, konnten sich Klebstoffe bis zum heutigen Tage noch nicht durchsetzen. Weiterhin werden Frakturen mit Platten-, Schrauben- und Nagelosteosynthesen stabilisiert und die Weichteile mit Nähten und Klammern versorgt. Dabei würde ein bioresorbierbarer Knochenkleber gegenüber den bekannten Verfahren entscheidende Vorteile bieten.

Ein Klebstoff verbindet getrennte Materialien schnell und einfach. Er wirkt sich auf die gesamte Oberfläche aus und sichert somit im Gegensatz zur Osteosynthese eine gleichmäßige flächenhafte Kraftverteilung. Außerdem kann der Kleber gleichzeitig alle Materialunebenheiten ausgleichen und eine Schwingungsdämpfung bewirken [19]. Ein anderer wichtiger Vorteil ist die Verminderung bzw. Vermeidung von Stress und Gewebeschäden in der Knochenbruchheilung bei der Verwendung von elastischem Kleber anstelle von metallischen Implantaten mit hoher Rigidität und Steifigkeit [39]. Ebenfalls ist die Blutversorgung beim Gebrauch von Klebstoffen unbceinträchtigt, während Schrauben- und Plattenosteosynthesen zu Durchblutungsstörungen und Gewebeirritationen führen können. Eine häufige Komplikation der Knochenbruchbehandlung durch eine Osteosynthese stellt die Metallose dar. Nahezu $5 \%$ aller Osteosynthesen haben heute eine Metallose zur Folge, die zu einer Instabilität und zur Frakturheilungsstörung führen kann.

Zum anderen muss ein herkömmliches Implantatmaterial durch eine Re-Operation entfernt werden. Damit steht der
Patient ein zweites Mal vor allen Risiken und Gefahren eines operativen Eingriffes. Dieser operative Eingriff und der damit verbundene Arbeitsausfall, wie auch die Operation zur Materialentfernung, bedeuten zusätzliche Kosten für das Gesundheitswesen.

\section{Einteilung der Klebstoffklassen}

Das Kleben ist eine der einfachsten und ältesten Fügetechniken, die der Mensch kennt. Als älteste Klebstoffanwender werden die Menschen der jüngeren Steinzeit genannt. Sie bedienten sich bereits vor über 7000 Jahren eines Birkenharz-Bienenwachs-Gemisches, um Pfeil und Speerspitzen zu bekleben [33]. Die Urbevölkerung benutze außerdem Naturklebstoffe aus Blut und Eiweiß [28]. Auch die alten Ägypter klebten mit Baumharzen, Eiweiß und tierischen Leimen aus Haut und Knochen [23].

Synthetisch hergestellte Klebstoffe gibt es seit 1870 [28]. Die unterschiedlichen Kleberarten können in zwei große Stoffklassen zusammengefasst und unterteilt werden (Tab.1). Zum einen gibt es die "biologischen körpereigenen oder körperähnlichen Klebstoffe", zum anderen die "synthetisch hergestellten körperfremden Adhäsive" (Tab.1). Die biologischen Adhäsive werden allerdings heutzutage auch synthetisch und industriell hergestellt, aber vom Ursprung her werden sie weiterhin als "biologisch" bezeichnet.

Der erste Versuch einen Kleber zu entwickeln wurde vor mehr als 4000 Jahren von den Ägyptern unternommen. Im Jahre 1772 wurde der "Plaster of Paris", der Gipsverband eingeführt, der heutzutage immer noch als konservatives Mittel zur Frakturbehandlung eingesetzt wird [39]. 1931 stellte HENDRI den ersten Knochenklebstoff als "Neues Prinzip der Osteosynthese" vor [22]. Der sterile Knochenleim Os- 
socol ${ }^{\circledR}$, ein aus Kollagen von Knochen und Bindegewebe gewonnenes Proteingemisch, zeigte anfänglich eine gute Klebefestigkeit. Starke lokale und allgemeine Reaktionen wie Schüttelfrost und mehrtägiges Fieber, das auf die antigen wirkenden xenogenen Proteine zurückzuführen war, verhinderten jedoch den weiteren Gebrauch [19].

Tab. I : Einteilung der Klebstofiklassen - Übersicht biologische und synthetische Klebstoffe

\begin{tabular}{|l|l|}
\hline Biologische Klebstoffe: & $\begin{array}{l}\text { Gelatine-Resorcin-Aldehyd-Klebstoffe } \\
\text { Protein-Aldehyd-Systeme } \\
\text { Fibrinklebstoffe } \\
\text { Klebstoffe auf Peptidbasis (Peptoplaste) } \\
\text { Resorbierbare Oligo-Polylactone }\end{array}$ \\
\hline Synthetische Klebstoffe: & $\begin{array}{l}\text { Epoxidharze } \\
\text { Polyurethane } \\
\text { (Alkyl-)Cyanoacrylate } \\
\text { Polymethylmethacrylate (PMMA) }\end{array}$ \\
\hline
\end{tabular}

1966 wurde der Gelatine-Resorcin-Aldehyd-Gewebeklebstoff, ein Gemisch aus Gelatine und Resorcin, das mit Formaldehyd gehärtet wird, entwickelt. Dabei reagieren Resorcin und Formaldehyd zu einem dreidimensionalen Netzwerk, während Gelatine als Füllstoff dient. Dieses Gemisch wurde experimentell und klinisch an der Haut, an Gefäßen und parenchymatösen Organen eingesetzt. Eine aufwendige Zubereitung und eine histotoxische Reaktion sprachen jedoch gegen weitere Versuche [19]. Später wurde das Formaldehyd durch die weniger toxischen Dialdehyde (Glutaraldehyd, Glyoxal) ersetzt. Diese Klebstoffkombinationen werden heutzutage vor allem in der Rekonstruktion von Aortenklappen und der Behandlung von Aortendissektionen sowie der Abdichtung chirurgischer Nähte verwandt [17].

Dagegen werden bei den Protein-Aldehyd-Systemen Proteine direkt als reaktive, vernetzbare Komponenten eingesetzt. Als Anwendungsbereich für solche Systeme wird z. B. die Beschichtung bzw. Primärabdichtung künstlicher Gefäßprothesen diskutiert. Seit 1998 wird in Europa auch ein chirurgischer Klebstoff (BioGlue ${ }^{\circledR}$ ) vertrieben, der zur Unterstützung der chirurgischer Hautnaht und bei der Behandlung von Aortendissektionen eingesetzt werden kann.

Die gegenwärtigen Fibrinklebstoffe werden in ihrer Form seit Beginn der 70er Jahre eingesetzt und stellen momentan den am weitest verbreiteten Gewebeklebstoff dar. Dabei handelt es sich um einen Zweikomponentenklebstoff. Eine Komponente besteht aus der Lösung einer Humanplasmaproteinfraktion mit Fibrinogen, Fibronectin, dem Blutgerinnungsfaktor XIII, Plasminogen sowie bovinem Aprotein als Fibrinolyseinhibitor. Die zweite Komponente ist eine Lösung aus Thrombin mit Zusätzen von Calciumchlorid. Der erste erfolgreiche Versuch Fibrin anzuwenden gelang PASSL et al. 1976 [34]. Bei Schafen wurden homologe Gelenkknorpeltransplantate ohne subchondrale Knochenplatte mit Fibrin und einem Cyanoacrylat fixiert. Gute Ergebnisse wurden nur an den Tieren erreicht, an denen das Gelenk postoperativ nicht ruhig gestellt wurde. Der Fibrinkleber beschleunigte bei den Knochentransplantaten und der kortikalen Frakturheilung die Knochenheilung und erhöhte die Rate der Revaskularisation [2, 6]. Weil der Klebstoff aus körpereigenen Substanzen besteht, ist seine Verträglichkeit hervorragend. Der Abbau des Fibrinklebstoffes geht im Gegensatz zu den nachfolgenden Klebstoffarten mühelos ohne größere Gewebereizung in wenigen Tagen von statten, welches für den Knochen aber in vielen Fällen zu kurz sein könnte [19].

In der heutigen Gewebeklebung finden Fibrinklebstoffe zur Unterstützung der chirurgischen Naht oder deren Einsparung in mechanisch gering belasteten Bereichen, z.B. dem Verschluss von Läsionen an inneren Organen wie Milz oder Leber Anwendung. Außerdem können sie zur Blutstillung, der Fixierung von Transplantaten im dermalen und HNO-Bereich, der Verklebung spannungsfreier Nervenanastomosen und der Verbesserung der Wundheilung eingesetzt werden. Für Klebungen, die mechanischen Beanspruchungen unterliegen oder über einen längeren Zeitraum stabil sein müssen, eignen sich diese Klebstoffe in der Regel nicht.

Klebstoffe auf Peptidbasis bieten sich als natürliche, im Organismus vorkommende Substanzen hinsichtlich ihrer Bioverträglichkeit und Abbaubarkeit für die Herstellung resorbierbarer medizinischer Klebstoffe besonders an. Die meisten natürlichen Systeme aus dem Tier- und Pflanzenreich sind außerordentlich komplex und daher kaum für die industrielle Herstellung geeignet. 1991 synthetisierte Rimpler et al. aushärtende Zweikomponenten-Klebstoffsysteme, sogenannte Peptoplaste, auf der Basis acrylierter Aminosäuren und Peptide [4]. Im Tierversuch zeigten diese Klebstoffe bei der Verklebung von kleineren Knochenfragmenten gute Ergebnisse, die Klebefestigkeit und Verträglichkeit wiesen aber eine unvollständige Degradation auf [37].

Eine andere Entwicklungsrichtung orientiert sich an marinen Organismen. Marine Weichtiere und Würmer bilden Klebstoffe auf Proteinbasis, die im Meerwasser bei unterschiedlichen Temperaturen aushärten und erstaunliche Klebefestigkeiten auf verschiedenen Materialien aufweisen. Auch hier stieß man auf einen kaum vertretbaren Aufwand in der Herstellung. Die modernen Methoden der Gentechnik könnten hier jedoch in nächster Zukunft eine Alternative bieten. Es ist bereits gelungen, den genetischen Code für die Herstellung von Wiederholungssequenzen der Peptidklebstoffe in Mikroorganismen wie E. coli zu übertragen und diese Bakterien zur Produktion der jeweiligen Sequenzen anzuregen, die dann in Gegenwart von Oxidasen tatsächlich Vernetzungsreaktionen eingehen [27].

Resorbierbare Polylactone, vor allem Polylactide, Polyglycolide und deren Copolyester haben in jüngster Zeit als "bioresorbierbare Osteosynthese" (Pins, Schrauben, Platten) sowie als Trägermaterialien für Pharmaka wachsende Aufmerksamkeit erlangt. In der Regel besitzen Polylactone eine freie terminale Hydroxylgruppe, die für Modifizierungen, u.a. zur Einführung vernetzungsfähiger Gruppierungen geeignet ist und die Herstellung von Klebstoffen auf Polylacton-Basis ermöglicht. Die Aushärtung zu einem Hydrogel wird photochemisch durch UV-Bestrahlung vorgenommen. Seit 1998 wird das Produkt in Deutschland vertrieben und hauptsächlich zum Verschluss von Luftleckagen nach Lungenoperationen verwendet, wobei der Klebstoff zusätzlich zu den konventionellen Techniken (Naht, Klammern) eingesetzt werden kann. Ebenfalls photochemisch härtbare acrylatterminierte Oligolactat- bzw. Glycolid-Systeme wurden für Anwendungen als Beschichtungen in der Dentaltechnik entwickelt.

Ende der 50er Jahre traten erstmalig synthetisch hergestellte Epoxidharze als Klebstoffe in den Vordergrund, die bereits in der Industrie zum Kleben, als Gießharze und Lacke verwendet wurden. Epoxidharze sind duroplastische Kunstharze, die durch Reaktion von Epichlorhydrin mit aromatischen Dihydroxyverbindungen entstehen [19]. 1958 versuchte BLOCH mit Araldit ${ }^{(8)}$, einem Kunststoffkleber aus Ethoxylinharzen, Frakturen an Vorderläufen von Schafen zu kleben. 
In 8 Fällen versagte die Methode infolge von auftretenden Infektionen oder weil sich das Milieu der Fraktur zu fetthaltig und zu feucht zeigte [5]. 1956-59 erzielte GOLOVIN gute Ergebnisse mit Osteoplast ${ }^{\circledR}$ an 32 Patienten mit frischen und pathologischen Frakturen sowie Pseudarthrosen. Zur Stimulierung der Kallusbildung mischte er den Klebstoff mit Zusätzen von Knochenmehl und Fibrin [21]. Nach Untersuchungen von GIEBEL et al. erreichte die Zugfestigkeit nach Applikation von Osteoplast ${ }^{\circledR}$ im feuchten Milieu nur 1/7 der normalen Knochenbiegefestigkeit [19].

Besonders RIETZ konnte 1964 durch seine Versuche beweisen, dass Epoxidharze nachfolgende Schwächen aufweisen [36] :

- Mangelnde Biokompatibilität

- Schlechte Degradation und Frakturheilungsstörung

- Hohe Polymerisationshitze beim Kleben

- Ungenügende Haftung des Klebestoffes am Knochen aufgrund eines feuchten Milieus

1959 lag eine sehr große Hoffnung in dem Polyurethan-Hartschaum Ostamer( Zwei Komponenten wurden gemischt, Kohlendioxid freigesetzt und eine Polyurethanverbindung mit 7-10\% Hohlraumbildung entstand. Diese Substanz erlangte nach $30 \mathrm{~min}$. seine Aushärtung und erreichte nach 18-24 h ihre maximale Festigkeit. Die experimentellen Untersuchungen zeigten jedoch eine Gewebstoxizität, Degradationsstörungen, Fragmentnekrosen und eine Hemmung der Osteoneogenese $[10,20]$. Des weiteren konnte eine ausbleibende oder geringe Kallusbildung $[10,30]$, lokale Wundheilungsstörungen und Infektionen mit Dehiszenzen, Hautnekrosen und Fistelungen beobachtet werden $[9,16]$. Ebenfalls konnte ein Verlust der Adhäsion im feuchten Milieu und eine hohe Polymerisationstemperatur von über $70^{\circ} \mathrm{C}$ aufgezeigt werden $[19,25]$. Diese Ergebnisse führten zu einem bis heute noch anhaltenden Vorurteil in der "American Orthopedic Community" gegenüber einer adhäsiven Osteosynthese.

1959 wurde durch Zufall von COOVER et al. die hohe Klebekraft von Cyanoacrylaten entdeckt [15]. Diese Klebstoffe, die auch in feuchter Umgebung gut binden, wurden schon in der experimentellen Chirurgie verwendet. Sie sind als Sekunden- und Sofortkleber in der Bevölkerung bekannt. Cyanoacrylate bestehen aus Estern der Acrylsäure und kalthärtendem Einkomponentenklebstoff. Eine Vielzahl von nützlichen Zwei-Cyanoacrylestern können durch Variation der Alkylkettenlänge synthetisiert werden. Die Elastizität und die Polymerisationszeit nehmen mit steigender C-Atomzahl der Alkylreste zu. Die Klebefestigkeit und die Toxizität der Verbindungen nehmen dagegen mit steigender C-Atomzahl ab $[14,18]$. Nachteilig für die Knochenfixation ist die geringe Fähigkeit der Cyanoacrylate kleine Spalträume aufzufüllen [7]. Die Knochenklebeversuche mit Cyanoacrylaten waren meist erfolglos. Wegen den regelmäßig auftretenden Dislokationen und einer in vitro nur $1 / 10$ der Biegebelastbarkeit des gesunden Knochens, rät GIEBEL von dem Gebrauch der Cyanoacrylate ab [18]. BECK empfiehlt kleinere Fragmente bei Gelenkfrakturen, die anders nicht mehr fixiert werden können, mit Cyanoacrylaten zu fixieren [3]. Biobond ${ }^{\circledR}$, ein Gemisch aus Ethylcyanoacrylat, Polyisocyanat und Nitril-Gummi, wurde 1979 zunächst hochgelobt, wegen der hohen Gewebetoxizität der kurzen Alkylketten wurden jedoch alle Applikationen gestoppt [31]. Eine gewisse karzinogene Wirkung wurde zeitweise diskutiert. Schließlich wurden die Methylcyanoacrylate von den lebensmitteltechnischen und pharmazeutischen Behörden für den menschlichen Gebrauch verboten.

Weitere Studien mit Bucrylaten, Butyl- und Isobutylcyanoacrylaten konnten keine karzinogene Wirkung bestätigen.
Sie zeigten zwar eine eher gutartige Gewebereaktion, aber da die Wirkstoffe bis zu vier Monaten im Gewebe verblieben, stellten sie eine Barriere für die Kallusformation dar. Cyacrin $^{\circledR}$ (Ethylcyanoacrylat) wurde in Osteuropa 1963 zunächst an Tieren getestet. Wegen guter Bioadhäsion und Frakturheilung wurden klinische Versuche an offenen und geschlossenen diaphysären Frakturen, Rippen-, Phalangealsowie Metacarpalfrakturen durchgeführt. TKACHENKO und RUTSKI berichteten von einer durchgängigen Biodegradation, einer guten Heilung und einer minimalen Gewebereaktion.

1972 veröffentlichte POLJAKOW ein Verfahren, bei dem ein Cyanoacrylat-Knochenmehlgemisch mit Ultraschall zur Sofortpolymerisation gebracht und in ein hartes Knochenkonglomerat verwandelt wurde [35]. Angeblich sollte die Verbindung dabei mit dem Knochenkollagen verschmelzen, weshalb er von einem "Knochenschweißen" sprach. In Tierversuchen und späteren klinischen Studien an diaphysären und intraartikulären Frakturen berichtete POLJAKOW von außerordentlich guten Ergebnissen. Mehrere Arbeitsgruppen bewiesen, dass sich mit dieser Art von Knochenklebung mit Hilfe einer Klebstoffmanschette eine primäre 50 \%ige Biegefestigkeit des intakten Knochens erreichen lies, aber durch die starke Wärmeentwicklung sich Osteonekrosen bildeten [8]. Weitere Nachteile wie hohe Infektionsraten, schwere lokale Toxizität, schlechte Resorption, fehlender Zusammenschluss und verschobene, nicht in ihrer geklebten Position verbliebene Frakturenden, konnten ebenfalls nachgewiesen werden.

Polymethylmethacrylate (PMMA) sind seit 1930 in der Zahnmedizin vertreten. 1965 versuchten CHARNLEY und KETTLEWELL Polymethylmethacrylat als erste für die Verankerung der Hüft-Total-Endoprothese zu verwenden [12]. Die Gewebereaktion zeigte eine minimale benigne Fremdkörperriesenzellreaktion [1, 11]. Außerdem wurde eine Polymerisationshitze mit einer Wärmespitze von $96^{\circ} \mathrm{C}$ ermittelt. Palacos, ein Methylmethacrylatpräparat, ist ein schnellhärtender, thermoplastischer Polymerisationskunststoff. Die biologische Verträglichkeit von Palacos wurde durch HULLINGER 1962 an Gewebekulturen geprüft [26], während ZOLLINGER histopathologische Untersuchungen vornahm [40]. HULLINGER zeigte, dass das ausgehärtete Methacrylat von Zellen sehr gut vertragen wurde, allerdings das noch flüssige Gemisch die Zellen abtötete. Diese zytotoxische Wirkung wurde auf das Monomer zurückgeführt. ZOLLINGER fand in der Umgebung von Palacos bei Sektionen 6 und 12 Monate nach dem Eingriff weder Abbau noch reaktive Erscheinungen wie Bildung von Granulationsgewebe. Daher bezeichnete er das Präparat, genau wie HOPPE [24], als ausgesprochen gewebefreundlich [32]. In Versuchen PMMA für die innere Knochenfixation zu verwenden, wurde beobachtet, dass PMMA keinen richtigen Klebstoff darstellte, aber mit spongiösem Knochen eine gute Verbundfestigkeit einging [38]. In Tierversuchen wurde PMMA als externe Manschette, als intramedulläre Schienung und als Klebstoff direkt in dem Frakturspalt erprobt. Alle Arbeitsgruppen berichteten von einer guten Fixationsstärke und adäquater Frakturheilung. Während gute Ergebnisse in Unterkiefer-, Rippen- und Schaftfrakturen verzeichnet wurden, sprachen späte Dislokationen und eine fehlende Klebung gegen den Gebrauch als Knochenklebstoff [13].

Derzeit werden neuartige bioresorbierbare Knochenkleber auf der Basis von Alkylen-bis(oligolactoyl)methacrylat (Firma Biomet Merck BioMaterials, Darmstadt) entwickelt und sowohl in vitro als auch im Tiermodell getestet (Abb. 1). Dieses neue Adhäsivsystem lässt sich keiner der zuvor be- 


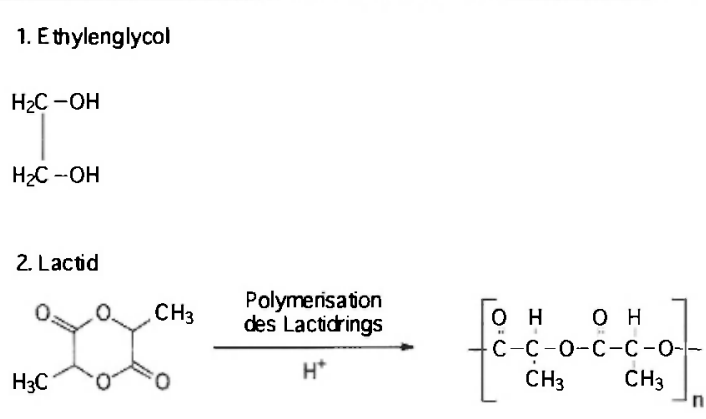

3. Methacrylsăure

$\mathrm{CH}_{3}$

$\mathrm{HOOC}-\mathrm{C}=\mathrm{CH}_{2}$

4. Ethylenglycol-oligolactid-bismethacrylat

$$
\begin{aligned}
& {\left[\begin{array}{llllll}
\mathrm{O} & \mathrm{CH}_{3}
\end{array}\right] \mathrm{O} \quad \mathrm{CH}_{3}} \\
& \mathrm{H}_{2} \mathrm{C}-\mathrm{O}-\mathrm{C}-\mathrm{C}-\mathrm{O} \cdot \mathrm{C}-\mathrm{C}=\mathrm{CH}_{2} \\
& \mathrm{H}]_{n} \\
& {\left[\begin{array}{ll}
\mathrm{O} & \mathrm{CH}_{3}
\end{array}\right] \mathrm{O} \quad \mathrm{CH}_{3}} \\
& \mathrm{H}_{2} \mathrm{C}-\mathrm{O}-\underset{\mathrm{C}}{\mathrm{C}}-\underset{\mathrm{H}}{\mathrm{C}}-\mathrm{O}-\underset{\mathrm{m}}{\mathrm{C}}-\stackrel{\mathrm{C}}{\mathrm{C}}=\mathrm{CH}_{2}
\end{aligned}
$$

Abb. I : Ein neuentwickelter bioresorbierbarer Knochenklebstoff - Zweistufige Synthese von Ethylenglycol-oligolactid-bismethacrylat (ELAMA), ausgehend von Ethylenglyckol (1), Lactid/Milchsäure (2) und Methacrylsäure (3).

schriebenen Klebstoffklassen eindeutig zu ordnen. Am besten kann dieser Knochenklebstoff aufgrund der Methacrylsäure als Grundbaustein zu den Polymethylmethacrylaten zugeordnet werden. Durch den zweiten Baustein der Milchsäure, die auch im menschlichen Körper vorkommt ist natürlich auch eine biologische Komponente vorhanden. Dies entspricht in etwa dem Mehrkomponentensystem des Gelatine-Resorcin-Aldehyd-Klebstoffes (Gelatine als biologischer und Resorcin-Aldehyd als körperfremder Baustein). Aus diesem Überblick der biologischen und synthetischen Klebstoffe ist ersichtlich, dass jeder Klebstoff, der nicht einer Degradation unterliegt, in einem nicht akzeptabelen hohen Prozentsatz zu einer fehlenden Verbundfestigkeit und Osteogenese (non-union) führt. Nur die mit langen Alkylketten bestückten Cyanoacrylate, die Fibrinklebstoffe und Polymethylmethacrylate scheinen gut vom Gewebe toleriert zu werden und zeigen somit eine gute Biokompatibilität.

\section{Anforderungen an einen bioresorbierbaren Knochenkleber}

Ein Knochenklebstoff kann eine einfache und schnelle Methode darstellen, um Abriss- und Kleinfragmentfrakturen ohne zusätzliches Osteosynthesematerial zu fixieren. Um den Anforderungen in der operativen Traumatologie und Orthopädie genügen zu können, sollte ein bioresorbierbarer Klebstoff die nachfolgenden Kriterien erfüllen (Tab. 2).

Zunächst muss die Klebeverbindung so stabil sein, dass sie möglichst sofort eine funktionelle Beanspruchung erlaubt
ORIGINAL

\begin{tabular}{|l|l|}
\hline Biohistokompatibilität & $\begin{array}{l}\text { nicht toxisch, nicht karzinogen, } \\
\text { nicht teratogen, nicht embryotoxisch } \\
\text { bioresorbierbar (degradierbar) } \\
\text { in einem definierten Zeitraum } \\
\text { geringe Polymerisationshitze } \\
\text { während der Aushärtungsphase }\end{array}$ \\
\hline Verbundfestigkeit & $\begin{array}{l}\text { starke Klebefähigkeit } \\
\text { ausreichende Elastizität } \\
\text { gutes Haftvermögen an feuchten, } \\
\text { fettigen und spongiösen Oberflächen }\end{array}$ \\
\hline Handhabung & $\begin{array}{l}\text { einfache Anwendbarkeit und Applizierbarkeit } \\
\text { Weitere Eigenschaften }\end{array}$ \\
\hline $\begin{array}{l}\text { schnelle Aushärtung } \\
\text { keine Volumenänderung } \\
\text { Stabilität während der Lagerung } \\
\text { Sterilisierbarkeit } \\
\text { Wirtschaftlichkeit }\end{array}$ \\
\hline
\end{tabular}

Tab. 2 : Allgemeine Anforderungen an bioresorbierbare Knochenklebstoffe in der operativen Traumatologie und Orthopädie

und sollte so lange bestehen bleiben, bis das Gewebe infolge einer physiologischen Frakturheilung diese Funktion wieder selbst übernehmen kann. Knochenklebstoffe sind nur brauchbar und anwendbar, wenn sie initial eine ausreichend starke Bindung für eine provisorische oder endgültige Fixation gewährleisten können. Zum Zeitpunkt der erfolgten knöchernen Heilung sollte der Klebstoff ohne Schaden resorbiert, auf der Haut aufgelöst oder abgestoßen sein [19]. Ziel eines jeden Eingriffs sollte die völlige Wiederherstellung der knöchernen Feinstruktur sein, welches die Grundlage einer langfristigen Stabilität darstellt. Der Knochenkleber muss deshalb vollständig degradierbar sein und mit körpereigenem Knochen ersetzt werden können. Die physiologische Knochenheilung muss während der Präsenz des Knochenklebers möglich sein, der keine Barriere darstellen sollte. Der Klebstoff muss ausreichend elastisch und zähflüssig sein, um von seinem Applikationsort nicht weichen zu können. Außerdem dürfen weder lokal- oder systemtoxische, noch mutagene, noch karzinogene oder embryotoxische $\mathrm{Ne}-$ benwirkungen nachweisbar sein, um so eine hohe Biokompatibilität gewährleisten zu können. Der Kleber sollte leicht sterilisierbar sein und auch unter operativen Bedingungen, wie z.B. in feuchter Umgebung und an fettigen Oberflächen wie spongiösem Knochen, fest haften. Bei der Applikation sollten keine größeren Temperaturen (Polymerisationshitze) entstehen, um Wärmeschäden im Gewebe (Hitzenekrosen) zu vermeiden. Eine einfache Anwendung und Umsetzung ist wünschenswert, damit jeder chirurgisch-orthopädisch tätige Arzt ohne zusätzliche Schulung den Knochenkleber erfolgreich nutzen kann. Schließlich muss sich der Knochenkleber in seiner Herstellung, Aufbewahrung und Anwendbarkeit als wirtschaftlich erweisen.

\section{Einsatzmöglichkeiten des Knochenklebers}

Die Beobachtungen und Ergebnisse, die in durchgeführten Kleintier- und Großtierexperimenten gewonnen wurden, sollen dazu beitragen, die Versorgung von Frakturen beim Menschen in Zukunft zu optimieren und die Einsatzgebiete eines Knochenklebers festzulegen. Besonders bei Trümmerfrakturen in Verbindung mit einer Osteosynthese und zur Refixation von kleinen Knochenfragmenten bei Frakturen oder Abrissfrakturen ohne zusätzliche osteosynthetische 
Maßnahmen dürfte durch einen Knochenkleber eine verbesserte Knochenheilung bewirkt werden. Vor allem die mäßig belasteten und beanspruchten Knochen im Fuß- und Handbereich dürften für die Kleberanwendung sehr interessant sein.

In anderen operativen Fachgebieten, wie der Mund-KieferGesichts-, der Neurochirurgie und Orthopädie dürfte bei der Versorgung der überwiegend filigranen Knochenbrüche oder bei elektiven Eingriffen die Anwendung des Knochenklebers ebenso hilfreich sein. Ebenfalls könnte man sich auf dem Gebiet der Hals-Nasen-Ohrenheilkunde vorstellen, dass es auch hier zahlreiche Indikationen für den Knochenkleber gibt.

Es sind zwar noch keine Überlegungen und experimentelle Untersuchungen über das Klebeverhalten des Knochenklebers in Weichteilgeweben, z.B. als Indikation in der Allgemein- und Viszeralchirurgie, gemacht worden, allerdings könnte sich dieser Fachbereich in näherer Zukunft auch als mögliches Anwendungsgebiet herausstellen. Der Kleber könnte des weiteren als Träger für Medikamente benutzt werden, wie die heutigen bekannten antibiotikahaltigen Knochenzemente. Dies setzt jedoch eine Resorbierbarkeit und Biokompatibilität des Klebers gegenüber dem umliegenden Gewebe voraus. Bei der zementierten Verankerung von Implantaten kommen heutzutage antibiotikahaltige Knochenzemente wie z.B. der mit Gentamicin versetzte Refobacin-Palacos ${ }^{\circledR}$ zum Einsatz. Sowohl bei der Knochendefektbehandlung als auch in der Endoprothetik ist eine lokale antibiotische Behandlung sinnvoll, um bakterielle Infektionen zu verhindern, die zu ernsten Komplikationen führen können [29]. Antibiotic Impregnated Polymethyl-Methacrylate Perlen (AIPMMA) werden in der Humanmedizin seit den frühen 70ern für die Behandlung und Vorbeugung von Infektionen angewandt. Auch für das Zytostatikum Methotrexat wurden die Eigenschaften der Freisetzung bereits festgestellt. Der Knochenkleber könnte somit ein komplexes Therapiesystem darstellen, das bei der Osteomyelitis und bei Weichteilinfektionen eingesetzt werden könnte. Auch als Träger-Matrix für eine Low-Dose Therapie der Osteoarthritis könnte der Kleber in Zukunft eine große Rolle spielen.

\section{Schlussfolgerungen}

Das Kleben von Kleinfragmentfrakturen und Trümmerfrakturen (in Kombination mit einer Osteosynthese) könnte eine attraktive Alternative und Ergänzung zur heutigen 0steosynthese in der klinischen und experimentellen Unfallchirurgie und Orthopädie darstellen. Ein Kleber verbindet getrennte Materialien schnell und einfach ohne zusätzliche Hilfsmittel wie Schrauben, Platten oder Nägel. Operationen zur Entfernung eines Osteosyntheseimplantates (Re-0peration) würden überflüssig werden und alle Risiken und Komplikationen, die ein Re-Eingriff mit sich bringt, würden entfallen.

Derzeit gibt es keine aktuellen Studien, die sich mit dem Einsatz von Knochenklebern bei der Frakturheilung oder der Refixation von Kleinfragmenten befassen. Im Rahmen von Klein- und Grosstierversuchen können sich neue Erkenntnisse für eine verbesserte Frakturversorgung und Refixation von Fragmenten ergeben. Die notwendigen Grundlagen für die Anwendung eines Knochenklebers zur Optimierung der Behandlung von Frakturen und Knochendefekten müssen erarbeitet werden. Im Vordergrund steht dabei die Beurteilung der Biokompatibilität und der Verbundfestigkeit des einzelnen Knochenklebers.

Folgende Aspekte sind am experimentellen Tiermodell zu überprüfen: Beurteilung der Knochenformation und -resorp- tion nach Applikation eines Knochenklebers im Frakturspalt. Untersuchungen bzgl. der knöchernen Integration, der Bioaktivität und der Wiederherstellung der Knochenstruktur (Bone Remodelling) unter Anwendung eines synthetisch hergestellten Knochenklebers sind notwendig. Untersuchungen zur Zelltoxizität und Entzündungsreaktionen sowie die Analyse der Knochenkleberdichte, der Oberfläche (Bone Bonding), der Festigkeit und der Struktur der Knochenneubildung nach Applikation müssen durchgeführt werden.

\section{Abstract}

Glueing is an attractive technique to link divided materials as it is easy and rapid to perform. The wish of trauma and orthopedic surgeons for alternatives to osteosynthesis is reflected in the development of a variety of surrogates of synthetic or biological origin. Despite a longstanding history of research in this field up to now a clinically applicable alternative could not have been found on the field of bone glueing in trauma and orthopedic surgery. The following review article is meant to provide the reader with a critical overview of all the alternative resorbable adhesives. Aim of the article is to classify the different compounds recommended and to define the requirements of a bioresorbable bone glue. Finally recommendations for todays clinical use of the adhesives are presented. In addition, we want to point to the tremendous progress made on this subject, made possible by the joint effort of orthopedic surgeons and basic researchers.

\section{Literatur}

[1] Albreksson T., Linder L.: A method for short and long term in vivo study of the bone implant interface. Clin Orthop 159: 269,1981

[2] Arbes H., Bosch P., Lintner F., Salzer M.: First clinical experience with heterologous cancellous bone grafting combined with the fibrin adhesive system. Arch Orthop Trauma Surg 98: 183, 1981

[3] Beck H.: Kunststoffklebung mit Cyanoacrylaten an Sehnen und Knochengeweben. Langenbecks Arch klin Chir 316: 563-568, 1966

\section{[4] Berndt H. 0., Rimpler M.: Adhäsion 35: 23-27, 1991}

[5] Bloch B.: Bonding of fractures by plastic adhesives. J Bone Joint Surg 40 A: 804-812, 1958

[6] Bosch P.: Die Fibrinspongiosaplastik. Experimentelle Untersuchungen und klinische Erfahrung. Wien Klin Wochenschr (Suppl) 93: 1, 1981

[7] Brauer G. M., Kumpula J. W., Termini D. J., Davidson K. M.: Durability of the bone between bone and various 2-cyanoacrylates in an aqueous environment. J Biomed Master Res 13: 593, 1979

[8] Brug E., Braunsteiner E., v. Gemmern C.: Die Ultraschallverschweißung von Knochen. Chirurg 47: 555-558, 1976

[9] Buchner H.: Erfahrungen mit Polyurethanschaum (Ostamer) bei der Behandlung von Knochenbrüchen. Klin Med (Wien) 16: 264-288, 1961 


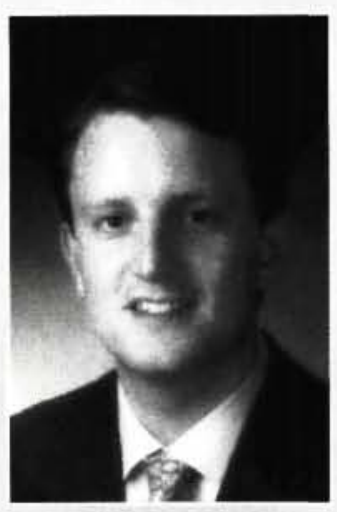

\section{Dr. med. Christian Heiss}

Dr. med. Christian Heiss

(Facharzt für Chirurgie)

Klinik und Poliklinik für

Unfallchirurgie, Justus-Liebig-

Universität Gießen

Rudolf-Buchheim-Strasse 7,

D-35385 Gießen

Tel.: 0641-9944200

Fax: 0641-9944609

Email:christian.heiss@chiru.

med.uni-giessen.de

Akademischer Lebenslauf

1990 - $1997 \quad$ Studium der Humanmedizin an der Johannes Gutenberg-Universität in Mainz

1997 Wissenschaftlicher Mitarbeiter des A0Research-Institute in Davos, Schweiz

1998 Promotion zum Dr. med. an der JustusLiebig-Universität in Gießen

$1998-1999$ Ausbildung als Arzt im Praktikum an der Klinik und Poliklinik für Unfallchirurgie der Justus-Liebig-Universität in Gießen

seit 1999 Assistenzarzt in der Klinik und Poliklinik für Unfallchirurgie der JustusLiebig-Universität in Gießen

1999 A0-Stipendium an der University of Mississippi, Medical Center, Orthopaedic Department, Jackson (USA)

1999 Preis der Deutschen Gesellschaft für Orthopädie und Traumatologie (DGOT) in Wiesbaden

2000 Verleihung des Harvard-Fellowships mit Aufenthalt am Massachusetts General Hospital, Harvard Medical School, Orthopaedic Hand Surgery Department, Boston (USA)

2003 Preis der ÖGU und GOTS auf dem Vienna Future Symposium in Wien, Austria

seit 2003 Facharzt für Chirurgie der Klinik und Poliklinik für Unfallchirurgie der Justus-Liebig-Universität in Gießen

[10] Buckner H., Feischl P.: Spätergebnisse bei der Behandlung von Knochenbrüchen mit Polyurethanschaum. Arch orthop Unfall-Chir 54: 48-57, 1962

[11] Cabanela M. E., Coventry M. B., Mac Carty C. S., Miller W. E.: The fate of patients with methyl- methacrylate cranioplasty. J Bone Joint Surg 54 A: 278, 1972

[12] Charnley J., Kettlewell J.: The elimination of slip between prosthesis and femur. J Bone Joint Surg 47 B: 56, 1965

[13] Charnley J.: The healing of human fractures in contact with self - curing acrylic cement. Clin Orthop 47: 157, 1966
[14] Contzen H.: Der derzeitige Stand der Gewebevereinigung durch klebende Autopolymerisate.

Melsunger Med Mitt 42: 7, 1968

[15] Coover H. W., Joyner F. B., Shearer N. H., Wicker T. H.: Chemistry and performance of cyanoacrylate adhesives. Soc Plastics Engrs J 15: 413, 1959

[16] Drompp B. W.: Chemical osteosynthesis of fractures and non-unions of the shafts of long bones of the lower extremity. Amer J Surg 99: 733-744, 1960

[17] Ennker J.: Gewebestoffe in der Thorax- und Kardiovaskularchirurgie. Steinkopff Verlag, Darmstadt 1994

[18] Giebel M. G.: Adhesives in surgery - Ergebnisse bei Klebungen mit verschiedenen Klebern an verschiedenen Geweben. Wien Med Akad 65-66, 1968

[19] Giebel G., Rimpler M.: Klebungen am Skelettsystem: Klebstoffe, 50 Jahre Hilfsstoffe für den Chirurgen (Teil 1). Biomed Techn 26: 35-40, 1981

[20] Gilmer W. S., Tooms R. E., Salvatore J. E.: An experimental study of the influence of implanted polyurethane sponges upon subsequent bone formation. Surg Gyn Obstetr 143-148, 1961

[21] Golovin G. V.: Frakturfixierung mit Kunststoff (Russisch). Vestn Khir Gekov 77: 125, 1956

[22] Hendri A.: Ein neues Prinzip der Osteosynthese. Arch Klin Chir 167: 145-146, 1931

[23] Historisch belegte Beiträge über das Zeitalter des Klebens. http://www.klebstoffe.com/Informationen/Geschichte. htm, 12.2002.

[24] Hoppe W.: Tierexperimentelle Untersuchungen über Gewebsreaktionen auf Injektionen von autopolymerisierendem Kunststoff. Dtsch Zahnärztl Z 11:837-847, 1956

[25] Hoyt W.: Diskussionsbeitrag. J Bone Joint Surg 42 A(5): 878,1960

[26] Hullinger L.: Untersuchungen über die Wirkung von Kunstharzen ("Palacos" und "Ostamer") in Gewebekulturen. Arch orthop Unfall-Chir 54: 504-512, 1962

[27] Kitamura M., Kawakami K., Nakamura, Tsumoto K., Uchiyama H., Ueda Y., Kumagai I., Nakaya T.: Expression of a model peptide of a marine mussel adhesive protein in Escherichia coli and characterization of its structural and functional properties. J Polym Sci A: Polym Chem 37: 729-736, 1999

[28] Klebstoff - Guide von Henkel. http://www.kurswerkstatt.de/knowhow/kleb/kleb().htm, 12.2002 .

[29] Kühn K.-D.: Knochenzemente für die Endoprothetik. Springer-Verlag, Berlin 2001

[30] Leemann R. A., Hedinger C., Jenny M.: Tierexperimentelle und histologische Ergebnisse bei der Frakturleimung mit dem Polyurethanpolymer "Ostamer". Schweiz Med Wschr 31: 908-914, 1961 


\section{ORIGINAL ARBEITEN}

[31] Meyer G., Muster D., Schmitt D., Jung P., Jaeger J. H.: Bone bonding through bioadhesives: Present status. Biomater Med Devices Artif Organs 7: 55, 1979

[32] Müller M. E.: Die Verwendung von Kunstharzen in der Knochenchirurgie. Arch orthop Unfall-Chir 54: 513-522, 1962

[33] Pantel B.: Klebstoffe im Bogenbau, das ABC der Verklebungen. http://webfarm3.milleniums.net:80...ualHostRoot/ wissen/kleb/kleb2_htm, 12.2002

[34] Passl R., Plenk H., Sauer G., Spängler H. P., Radaszkiewicz T., Holle J.: Die homologe reine Gelenkknorpeltransplantation im Tierexperiment. Arch orthop Unfall-Chir 86: 243-256, 1976

[35] Poljakow W. A.: Resultate und Ausblicke der Anwendung des Ultraschallverfahrens in der Traumatologie. Unfallmedizinische Tagung der Landesverbände der gewerblichen Berufsgenossenschaften 16: 85-91, 1972

[36] Rietz K. A.: Polymer osteosynthesis. Acta Chir Scand 128: 349-401, 1964

[37] Rimpler M.: 8. Internationales Symposium - "Leistungsfahigkeit der modernen Klebetechnik",

in: Swissbonding 94, Rapperswil, Tagungsband 161-175, 1994

[38] Smith D. C.: Medical and dental applications of cements. J Biomed Mater Res Symp 1: 189, 1971

[39] Wenz R.: First results with a bioresorbable bone glue, in: Biomaterials in Surgery, edited by GHîM Walenkamp, Thieme Verlag, Stuttgart 1998

[40] Zollinger H. U.: Experimentelle Erzeugung maligner Nierenkapseltumoren bei der Ratte durch Druckreiz (PlasticKapseln). Schweiz Z Allg Path 15: 666, 1952 